\title{
Association between lifestyle and thyroid dysfunction: a cross-sectional epidemiologic study in the She ethnic minority group of Fujian Province in China
}

Yanling Huang ${ }^{1,2+}$, Liangchun Cai ${ }^{2+}$, Yuanyuan Zheng ${ }^{2 \dagger}$, Jinxing Pan ${ }^{3+}$, Liantao Li ${ }^{2}$, Liyao Zong ${ }^{2}$, Wei Lin², Jixing Liang ${ }^{2}$, Huibin Huang ${ }^{2}$, Junping Wen ${ }^{2}$ and Gang Chen ${ }^{2,4^{*}}$

\begin{abstract}
Background: Thyroid dysfunction is one of the prevalent endocrine disorders. The relationship between lifestyle factors and thyroid dysfunction was not clear and some of the factors seemed paradoxical.

Methods: We conducted this population-based study using data from 5154 She ethnic minority people who had entered into the epidemic survey of diabetes between July 2007 to September 2009. Life style information was collected using a standard questionnaire. Body mass index (BMI), Blood pressure and serum TSH, TPOAb, triglycerides (TG), total cholesterol (TC) and high-density lipoprotein cholesterol (HDL) were collected.

Results: The study showed that people who drank, had higher education or suffered from insomnia have lower incidence of hyperthyroidism. On the other hand, smoking, alcohol consumption, exercise, undergoing weight watch and chronic headache were associated with decreased incidence of hypothyroidism. Using multivariable logistic regression analysis, we found that alcohol consumption was associated with decreased probability of hyperthyroidism, hypothyroidism, as well as positive TPOAb. The amounts of cigarettes smoked daily displayed a positive correlation with hyperthyroidism among smokers. Accordingly, smoking seemed to be associated with decreased risk for hypothyroidism and positive TPOAb. Exercise and maintaining a healthy weight might have a beneficial effect on thyroid health. Interestingly, daily staple amount showed an inverse correlation with incidence of positive TPOAb.
\end{abstract}

Conclusions: Within the Chinese She ethnic minority, we found associations between different lifestyle factors and the incidence of different thyroid diseases. Understanding the nature of these associations requires further investigations.

Keywords: Life style, Epidemiologic study, Hyperthyroidism, Hypothyroidism, Thyroid peroxidase antibody (TPOAb)

\footnotetext{
* Correspondence: chengangfj@163.com

†Yanling Huang, Liangchun Cai, Yuanyuan Zheng and Jinxing Pan

contributed equally to this work.

${ }^{2}$ Department of Endocrinology, Shengli Clinical Medical College of Fujian

Medical University, Fuzhou 350001, China

${ }^{4}$ Department of Endocrinology, Fujian Provincial Hospital, Fujian Academy of

Medical Sciences, Fuzhou 350001, China

Full list of author information is available at the end of the article
}

(c) The Author(s). 2019 Open Access This article is distributed under the terms of the Creative Commons Attribution 4.0 International License (http://creativecommons.org/licenses/by/4.0/), which permits unrestricted use, distribution, and reproduction in any medium, provided you give appropriate credit to the original author(s) and the source, provide a link to the Creative Commons license, and indicate if changes were made. The Creative Commons Public Domain Dedication waiver (http://creativecommons.org/publicdomain/zero/1.0/) applies to the data made available in this article, unless otherwise stated. 


\section{Background}

Thyroid dysfunction is one of the prevalent endocrine disorders. Thyroid dysfunction can lead to various health issues including cardiovascular disorders [1], abnormal glucose intolerance, impairment of liver function, dyslipidemia, and so on. Thyroid dysfunction may also cause psychological symptoms and affect quality of life [2]. It was found that even at subclinical level, hyperthyroidism and hypothyroidism are associated with overall reduction of medical well-being [3].

The observation of different prevalence rate among different ethnic groups and geological areas indicates that both genetic and environmental factors contribute to the development of thyroid dysfunction. It is well known that most of the thyroid dysfunction is caused by autoimmune thyroid diseases (AITD). Thus it is not surprising that polymorphisms in major histocompatibility genes (human leukocyte antigen [HLA]), immunoregulatory genes (CTLA4, PTPN22, FOXP3, CD25, CD40, $F R C L 3)$, and thyroid specific genes (TSHR,TG) were found to be associated with increased prevalence of the disease [4]. On the other hand, There are also indications that environmental factors such as alcohol consumption, smoking, Iodine intake, deficiency in vitamin and minerals such as Vitamin D and Selenium, infections, stress, and certain drugs (estrogens) may also affect the incidence of the thyroid dysfunction [5]. How different environmental and lifestyle factors affect development of thyroid disease is not well understood. Interestingly, recent studies have indicated that environmental and lifestyle factors may interact with genetic factors by influencing epigenetic mechanisms, such as altering DNA methylation and chromatin modifications, thereby modulating gene expression [6]. Studies from many areas around the world have investigated potential environmental risk factors of thyroid dysfunction. However, some of the results remain controversial. For example, a survey by Nystrom et al. (1993) identified smoking as a risk factor for hypothyroidism [7], while other studies failed to find any association [8]. A recent research in fact found that smoking might reduce the risk of hypothyroidism [9]. Besides smoking, other lifestyle factors need to be explored to identify additional contributors to the disease development.

We have the complete dataset of an epidemic survey of diabetes in the She ethnic minority group of Fujian Province in China, which was carried out from July 2007 to September 2009. The details of this study have been published previously [10-12]. Among the participants, 5154 were tested for the TSH and TPOAb levels. Using these data, we conducted statistical analyses to investigate potential associations between several lifestyle factors and the incidence of thyroid dysfunction.

\section{Methods}

\section{Study design and population}

The She ethnic minority is one of the smaller ethnic populations in China. Confining our study to such a cohort with relatively homogenous genetic and general environmental background allows us to better isolate lifestyle factors from the genetic components of the disease, as well as minimize the effect of other hidden environmental variables. The study is part of an epidemic survey of the diabetes in the She ethnic minority group of Fujian Province. We recruited participants by advertising and phone calls made by the community workers. Subjects registered were permanent residents of the Fujian province She reservation area, aged $20-80$ years. A multistage, stratified, cluster random sampling method was used in order to select a representative sample. A total of 5385 She ethnic residents were enrolled in the survey and 5154 subjects who had results of TSH and TPOAb levels were collected in our study. The study was approved by the Ethics Committee of Fujian Medical University. We had complied with the Declaration of Helsinki Ethical Principles for medical research involving human subjects [13]. All the participants signed informed consent authorized by the Chinese Medical Association before data collection.

\section{Patient and public involvement}

The role of subjects in this study were participants. They were not involved in the recruitment or conduction of the study. After the completion of the study, we sent each participant a letter describing the detailed results of their own physical examinations and lab tests free of charge. If the lab tests or physical examinations showed abnormal results, we informed the participants by phone calls and advised them to go to the clinic for appropriate further examination and treatments.

\section{Data collection}

Subjects were individually interviewed by trained staff. During the interview, life style information was collected using a standard questionnaire. Smokers were defined as those who were having or had had at least 5 cigarettes per day. The numbers of cigarettes taken by the smokers were also recorded. As the She minority group use cereal as their staple food, daily staple was defined as the amount of cereal consumed per day. Alcohol consumption was defined as the average consumption of at least $35 \mathrm{~g}$ of alcohol per day. Salt intake was an estimation of the individual's salt intake per year. Due to mandatory iodization of salt in the area, salt intake was a proxy for iodine intake. Exercise was self-reported, defined as performing physical activities for at least 20-30 min twice or more per week. Dietary restriction was defined 
as being on a low fat and low-calorie diet. Frequent sugary beverages referred to consuming sugary beverages more than twice a week. People who measured their body weight more than once a week were characterized as "yes" for weight watching. Chronic insomnia referred to having disrupted sleep that occurred at least three nights per week and lasting for at least three months. Chronic daily headache was defined as having a headache for more than four hours on more than 15 days per month. In addition to the above life style information, the accompany diseases and medication, family history of thyroid disease were also recorded. The participants who had already had thyroid disease were excluded.

\section{Physical examinations}

Body mass index (BMI) was calculated as weight in kilograms divided by height in meters squared $\left(\mathrm{kg} / \mathrm{m}^{2}\right)$. Blood pressure was measured twice in the sitting position by manual sphygmomanometer on the right arm after at least $5 \mathrm{~min}$ of rest, and the mean of the two readings was used for analysis. All of the staffs conducting the physical examinations completed a training program of the entire study protocol before they began to collect data.

\section{Laboratory measurements}

Serum TSH was assessed using an electrochemiluminescence (Nichols Institute Diagnostics, San Juan Capistrano, CA, USA). The working range for this method was 0.01$100 \mathrm{mIU} / \mathrm{L}$. Serum TPOAb were measured with a highly sensitive, direct RIA system (Kronus, San Clemente, CA, USA). The reference range of TPOAb was $\leq 35 \mathrm{IU} / \mathrm{ml}$. Serum triglycerides (TG). Total cholesterol (TC) and high-density lipoprotein cholesterol (HDL) were determined by an automatic colorimetric method (Hitachi; Boehringer Mannheim). LDL-cholesterol (LDL-c) was calculated using the Friedewald formula. All blood samples were obtained in a fasting state at $8 \mathrm{am}$.

\section{Diagnostic categories}

Globally, the normal range of TSH level is not definitely set. Different regions, ages and ethnic groups may have different ranges. To set a reference range for normal TSH values, we selected 250 healthy participants aged 20-80 and carefully excluded those who self-reported thyroid disease or goiter, as well as those who had thyroid disease family history. We tested their TSH levels and set the range of 2.5th- 97.5th percentile as the reference range of normal TSH values. The resulting range was $0.34 \mathrm{mIU} / \mathrm{L}-4.22 \mathrm{mIU} / \mathrm{L}$. TSH level lower than $0.34 \mathrm{mIU} / \mathrm{L}$ was defined as hyperthyroidism (including subclinical hyperthyroidism). TSH level higher than $4.22 \mathrm{mIU} / \mathrm{L}$ was defined as hypothyroidism (including subclinical hypothyroidism).

\section{Statistical analysis}

Statistical analyses were conducted using the SPSS software (version 13.0, SPSS, Chicago). The distributions of numerical variables were tested for normality. Data with normal distribution were expressed as means \pm standard deviation (SD). Data with skewed distribution were logtransformed for analysis. If still with skewed distribution, data were expressed as median (interquartile range). For categorical variables, data were expressed as percentages. Student's t-test was used for continuous variables and chi-square test for categorical variables to assess differences. Multiple logistic regression analyses were performed to evaluate factors associated with thyroid dysfunction. We set whether or not having thyroid dysfunction as the dependent variable (yes $=1$, no $=0$ ). The significant variables identified by previous Student's t-test or chi-square test were selected to be included in the regression analyses. Continuous variables such as BMI were classified by the value of clinical significance (eg. $\quad$ BMI < 18.5: underweight; 18.5 23.9:normal; 24 27.9:overweight; $\geq 28$ :obesity). After testing for all possible interactions among independent variables to eliminate the influence of confounders, the best fitted logistic regression model was established to determine the possible risk factors for thyroid dysfunction. Adjusted ORs and 95\% confidence intervals were calculated for all significant variables. $P<0.05$ was considered to be statistically significant.

\section{Results}

Out of a total of 5700 subjects initially surveyed by the questionnaire, we were able to obtain completed questionnaires with corresponding laboratory data for 5154 of them. The response rate was $90.4 \%$. The prevalence of thyroid dysfunction was shown in the Table 1.

Among these 5154 participants, based on TSH level, $140(2.72 \%)$ were diagnosed with hyperthyroidism (aged from 20 to 80 ) and 206 (4.00\%) were diagnosed with hypothyroidism (aged from 20 to 80). Incidence of TPOAb positive was $10.65 \%$. The characteristics of the subjects categorized by thyroid status were presented in Table 2.

As expected, compared with people with euthyroidism, those with hyperthyroidism had higher heart rates and lower TC and LDL level. People with hypothyroidism had higher BMI. The influence of thyroid disorders on UA level was currently unclear. In our study, people with hyperthyroidism and positive TPOAb both had lower UA level. Compared with TPOAb negative, people who were TPOAb positive had higher age, BMI, HR, and HDL level.

Table 3 showed life style factors and their associations with thyroid status. As shown in the table, we found hyperthyroidism occurring less frequently among people 
Table 1 Prevalence of thyroid dysfunction in the study

\begin{tabular}{llllll}
\hline & Subjects & Abnormal TSH n (\%) & Hyperthyroidism n (\%) & Hypothyroidism $\mathrm{n}(\%)$ & TPOAb positive $\mathrm{n}(\%)$ \\
\hline Male & & & & \\
$20-44$ & 941 & $35(3.7 \%)$ & $16(1.7 \%)$ & $19(2.0 \%)$ & $48(5.1 \%)$ \\
$45-59$ & 829 & $39(4.7 \%)$ & $23(2.8 \%)$ & $16(1.9 \%)$ & $62(7.5 \%)$ \\
$60-80$ & 452 & $39(8.6 \%)$ & $16(3.5 \%)$ & $23((5.1 \%)$ & $48(10.6 \%)$ \\
All males & 2222 & $113(5.1 \%)$ & $55(2.5 \%)$ & $58(2.6 \%)$ & $158(7.1 \%)$ \\
Female & & & & $67(5.0 \%)$ & $178(13.3 \%)$ \\
$20-44$ & 1339 & $103(7.7 \%)$ & $36(2.7 \%)$ & $55(4.5 \%)$ & $161(13.2 \%)$ \\
$45-59$ & 1217 & $93(7.6 \%)$ & $38(3.1 \%)$ & $26(6.9 \%)$ & $52(13.8 \%)$ \\
$60-80$ & 376 & $37(9.8 \%)$ & $11(2.9 \%)$ & $148(5.0 \%)$ & $391(13.3 \%)$ \\
All females & 2932 & $233(7.9 \%)$ & $85(2.9 \%)$ & $206(4.0 \%)$ & $549(10.7 \%)$ \\
All subjects & 5154 & $346(6.7 \%)$ & $140(2.7 \%)$ & & \\
\hline
\end{tabular}

who drank, had higher education or suffered from insomnia $(P<0.05$ by chi square test). Incidence of hypothyroidism was decreased among people who smoked, drank alcohol, exercised, underwent weight watch, or suffered from chronic daily headache $(P<0.05$ by chi square test). People who have higher education, smoke, consume alcohol, or undergo weight watch seem less likely to be TPOAb positive $(P<0.05$ by chi square test). We also analyzed daily cigarette intake and daily staple food consumption among normal individuals and individuals with thyroid dysfunction. As shown in Table 3, we found that compared to people with euthyroidism, people with hyperthyroidism smoke significantly more cigarettes daily $(P<0.01$ by student's t-test). In addition, we found people with positive TPOAb were those who consume less daily staple food.
Table 4 showed result of logistic regression analysis of the association of various lifestyle factors with hyperthyroidism, hypothyroidism and positive TPOAb. We set whether or not having thyroid dysfunction as the dependent variable (yes $=1$, no $=0$ ) and lifestyle factors with significant value in Student's t-test and chi-square test as independent variables for multiple unconditioned logistic regression analysis. For hyperthyroidism, alcohol consumption was found to be associated with decreased probability of disease incidence (OR $[95 \% \mathrm{CI}]=0.433$ [0.219-0.857]), whereas increased daily cigarettes consumption was found to be associated with increased risk, though the trend was weak (OR $[95 \%$ CI $]=1.045$ [1.019-1.073]). For hypothyroidism, exercise (OR [95\% $\mathrm{CI}]=0.678[0.467-0.984)]$ ), alcohol consumption (OR $[95 \%$ CI $]=0.522[0.351-0.775])$ and having chronic

Table 2 Characteristics of the subjects categorized by thyroid status

\begin{tabular}{llllll}
\hline & $\begin{array}{l}\text { Euthyroidism } \\
(n=4798)\end{array}$ & $\begin{array}{l}\text { Hyperthyroidism } \\
(n=140)\end{array}$ & $\begin{array}{l}\text { Hypothyroidism } \\
(n=206)\end{array}$ & $\begin{array}{l}\text { TPOAb negative } \\
(n=4605)\end{array}$ & $\begin{array}{l}\text { TPOAb positive } \\
(n=649)\end{array}$ \\
\hline Age (years) & $46.62 \pm 12.64$ & $49.17 \pm 11.43^{\mathrm{a}}$ & $48.00 \pm 14.42$ & $46.62 \pm 12.73$ & $47.88 \pm 12.23^{\mathrm{d}}$ \\
Sex (male/female) & $2109 / 2699$ & $55 / 85$ & $58 / 148^{\mathrm{c}}$ & $2064 / 2541$ & $158 / 391^{f}$ \\
BMI (Kg/m $\left.{ }^{2}\right)$ & $22.76 \pm 3.11$ & $22.32 \pm 3.15$ & $23.37 \pm 3.79^{\mathrm{a}}$ & $22.74 \pm 3.14$ & $23.04 \pm 3.18^{\mathrm{d}}$ \\
SBP (mmHg) & $134.19 \pm 23.29$ & $137.33 \pm 25.08$ & $136.67 \pm 27.27$ & $134.27 \pm 23.42$ & $135.49 \pm 24.02$ \\
DBP (mmHg) & $79.76 \pm 13.55$ & $80.77 \pm 13.54$ & $81.21 \pm 14.26$ & $79.80 \pm 13.45$ & $80.20 \pm 12.93$ \\
HR (bpm) & $74.33 \pm 14.20$ & $84.43 \pm 19.24^{\mathrm{c}}$ & $73.24 \pm 13.33$ & $74.27 \pm 14.14$ & $76.76 \pm 16.11^{\mathrm{e}}$ \\
TG (mmol/L) & $1.07(0.77-1.58)$ & $0.98(0.70-1.42)^{\mathrm{a}}$ & $1.12(0.76-1.68)$ & $1.07(0.77-1.59)$ & $1.05(0.75-1.49)$ \\
TC (mmol/L) & $4.95 \pm 1.38$ & $4.69 \pm 1.16^{*}$ & $5.08 \pm 1.17$ & $4.94 \pm 1.39$ & $4.99 \pm 1.08$ \\
HDL (mmol/L) & $1.48 \pm 0.33$ & $1.45 \pm 0.36$ & $1.51 \pm 0.32$ & $1.47 \pm 0.33$ & $1.50 \pm 0.34^{\mathrm{d}}$ \\
LDL-c (mmol/L) & $3.18 \pm 0.87$ & $3.00 \pm 0.89^{\mathrm{a}}$ & $3.28 \pm 0.98$ & $3.17 \pm 0.87$ & $3.23 \pm 0.89$ \\
UA (umol/L) & $280.58 \pm 81.57$ & $264.62 \pm 80.72^{\mathrm{a}}$ & $272.76 \pm 81.75$ & $281.46 \pm 80.90$ & $263.65 \pm 75.43^{f}$ \\
TSH (m/U/L) & $1.44(1.00-2.05)$ & $0.02(0.01-0.24)^{c}$ & $5.69(4.67-10.59)^{c}$ & $1.43(0.99-2.07)$ & $1.80(0.97-2.88)$ \\
TPOAb positive (\%) & 8.0 & $48.6^{c}$ & $36.4^{c}$ & &
\end{tabular}

$B M I$ body mass index, $S B P$ systolic blood pressure, $D B P$ diastolic blood pressure, $H R$ heart rate, $T G$ triglycerides, $T C$ total cholesterol, $H D L$ high-density lipoproteincholesterol, $L D L-c$ low-density lipoprotein-cholesterol, UA uric acid, Data shown as mean $\pm S D$ or $\%$. ${ }^{a} P<0.05$ when comparing with euthyroidism; ${ }^{b} P<0.01$ when comparing with euthyroidism; ${ }^{c} P<0.001$ when comparing with euthyroidism; ${ }^{\mathrm{d}} P<0.05$ when comparing with TPOAb negative; ${ }^{\mathrm{e}} P<0.01$ when comparing with TPOAb negative; ${ }^{f} P<0.001$ when comparing with TPOAb negative; TG and TSH are shown as median and interquartile range 


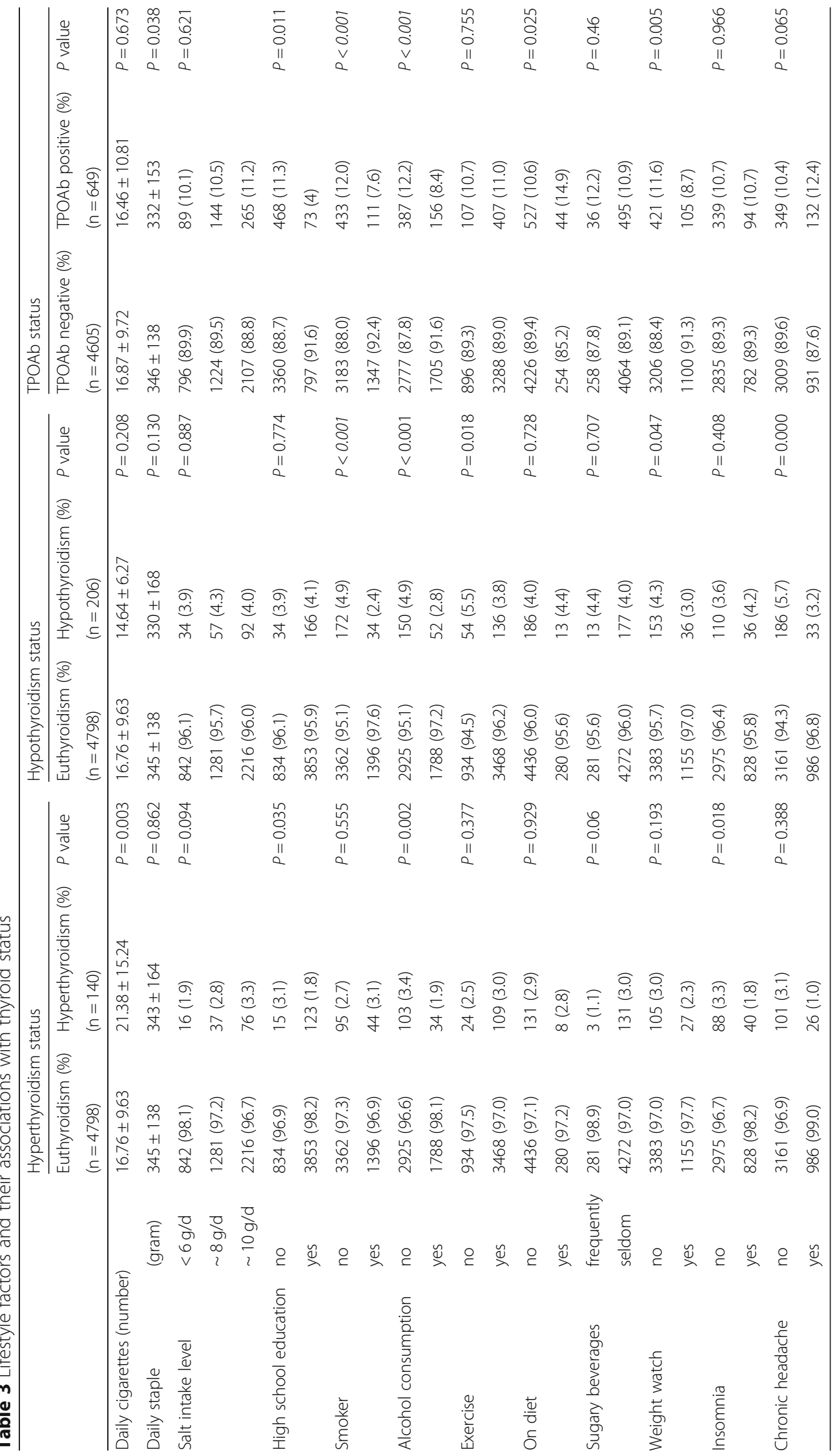


Table 4 Multivariate logistic regression analysis of risk factors of hyperthyroidism, hypothyroidism and positive of POAb

\begin{tabular}{lllll}
\hline & Factor & OR & OR $(95 \mathrm{Cl})$ & $P$ value \\
\hline Hyperthyroidism & alcohol consumption & 0.433 & $(0.219,0.857)$ & 0.016 \\
& daily cigarettes & 1.045 & $(1.019,1.073)$ & 0.001 \\
& insomnia & 2.234 & $(0.689,7.247)$ & 0.181 \\
& education & 1.247 & $(0.779,1.995)$ & 0.357 \\
& constant & 0.022 & & $<0.001$ \\
Hypothyroidism & BMl & 1.072 & $(1.020,1.126)$ & 0.006 \\
& alcohol consumption & 0.522 & $(0.351,0.775)$ & 0.001 \\
& exercise & 0.678 & $(0.467,0.984)$ & 0.041 \\
& chronic daily headache & 0.665 & $(0.469,0.943)$ & 0.022 \\
& smoker & 0.702 & $(0.443,1.112)$ & 0.131 \\
& weight concern & 0.770 & $(0.510,1.164)$ & 0.215 \\
& constant & 0.026 & & $<0.001$ \\
daily staple & 0.956 & $(0.918,0.995)$ & 0.028 \\
BMl & 1.030 & $(1.000,1.061)$ & 0.047 \\
alcohol consumption & 0.754 & $(0.606,0.937)$ & 0.011 \\
smoker & 0.758 & $(0.595,0.966)$ & 0.025 \\
weight watch & 0.730 & $(0.579,0.921)$ & 0.008 \\
high school education & 0.799 & $(0.627,1.020)$ & 0.071 \\
on diet & 0.784 & $(0.615,1.000)$ & 0.050 \\
constant & 0.147 & & $<0.001$ \\
\hline & & & &
\end{tabular}

headache $(\mathrm{OR}[95 \% \mathrm{CI}]=0.665 \quad[0.469-0.943])$ were found to be associated with decreased probability of developing the disease, whereas BMI (OR $[95 \% \mathrm{CI}]=1.072$ [1.020-1.126]) appeared to correlate positively with risk. When TPOAb positivity was analyzed, we found undergoing weight watch $(\mathrm{OR}[95 \% \mathrm{CI}]=0.730[0.579-0.921])$, smoker $(\mathrm{OR}[95 \% \mathrm{CI}]=0.758[0.595-0.966])$, or alcohol consumption $(\mathrm{OR}[95 \% \mathrm{CI}]=0.754[0.606-0.937])$ was each associated with decreased risk whereas BMI (OR $[95 \% \mathrm{CI}]=0.661 .030[1.000-1.061])$ was associated with increased risk. Interestingly, increasing daily staple amount appeared to correlate with decreased risk of positive TPOAb, although the effect is moderate (OR $[95 \% \mathrm{CI}]=0.956[0.918-0.995])$. The collinearity of the variables in the regression equations were assessed with collinearity diagnostics. The characters of Tolerance, Eigenvalue and Condition Index indicated that the variables in the three regression equations did not have multicollinearity.

\section{Discussion}

The epidemiological features of thyroid status in She ethnic minority

Among the 5154 subjects included in the research survey, the overall prevalence of thyroid dysfunction was $6.72 \%$ (with 2.72 and $4 \%$ for hyper- and hypothyroidism, respectively, as shown in the result section in Table 1). The TPOAb positive rate was $10.7 \%$ (Table 1). The trend was consistent with a recent large-scale epidemiological survey in China [14]. In male, there was no significant difference between the prevalence of hyperthyroidism and hypothyroidism. In female, the incidence of hypothyroidism and TPOAb positive were significantly higher than that of hyperthyroidism (Table 1). With the increase of age, the incidence of hyperthyroidism and hypothyroidism both increased in male, which was consistent with the survey in other countries [15].

\section{Alcohol consumption}

The effect of alcohol consumption on AITD is elusive. Some previous research found no association between alcohol consumption and Graves' Disease [16], while others suggested that moderate alcohol consumption might reduce the risk of Graves' disease [17]. Autoimmune thyroid disease seems to be much more dependent on environmental factors than hitherto anticipated. A populationbased case-control study in Denmark likewise observed that moderate alcohol consumption reduced the risk of overt autoimmune hypothyroidism [18]. In our study, alcohol consumption was found to be associated with reduced risk in hyperthyroidism, hypothyroidism and positive TPOAb (see Result section, Tables 3 and 4). The observed associations were independent of the type of alcohol (e.g. wine or beer), or gender. A protective effect of alcohol had also been reported for other autoimmune diseases such as rheumatoid arthritis, systemic lupus erythematosus and type 1 diabetes [19]. However, the mechanism of action of these protective effects of alcohol remains poorly understood.

\section{Smoking}

The association between smoking and autoimmune thyroid disease (AITD) was explored by many epidemiological studies. It was confirmed that smoking is a risk factor for the development of Graves' hyperthyroidism $(\mathrm{GH})$, especially for Graves' ophthalmopathy (GO) [20]. In consistence with these previous reports, our study also found smoking associated with increased incidence of hyperthyroidism. The association between smoking and hypothyroidism is less clear. According to a review by Vestergaard P. (2002), a number of previous studies failed to find any association between smoking and hypothyroidism [8]. However, several recent researches have provided strong evidence that smoking might reduce incidence of hypothyroidism [21, 22]. In our study, positive TPOAb was less prevalent in subjects who were smokers at the time of the study than it was in subjects who were not smoking $(7.6 \%$ vs. $12.0 \%, P<0.001)$. 
Similarly, hypothyroidism was less prevalent in smokers than it was in non-smokers $(2.4 \%$ vs. $4.9 \%, P<0.001)$. The mechanism of the protecting effect of smoking for hypothyroidism is unclear. Some research indicated that nicotine might play a role. Nicotine was thought to increase Tregs-mediated immune suppression of lymphocytes via $\alpha 7$ nicotinic acetylcholine receptor ( $\alpha 7 \mathrm{nAChR})$ [23]. Cigarettes smoking exposure was believed to inhibit Th1 cytokine production and may lead to enhancement of Th2 response [24]. Since Hashimoto's thyroiditis was regarded as predominantly a disease caused by increased Th1 production, while Graves's disease may be caused by the enhancement of Th2 response, the suppressive effect of cigarette smoking on Th1 response and the resulting enhancement of Th2 response may explain the decreased risk of hypothyroidism and increased risk of hyperthyroidism among smokers [25]. The effect of the smoking on the hyperthyroidism and hypothyroidism is interesting. Further research is required to better understand the underlying mechanisms.

\section{Exercise, weight watch and BMI}

Little has been done to examine potential impact of exercise and physical activities on thyroid function. Studies in healthy, well-trained male athletes had shown that short-term high intensity exercise increased levels of circulating thyroid hormones [26]. In contrast, for long-term exercise, a study of men after six months of endurance training reported that free $\mathrm{T} 4$ concentrations were slightly decreased, with no change in TSH [27]. In our study, we did not find any correlation between exercise and hyperthyroidism. However, in the case of hypothyroidism, the prevalence was lower in the subjects who exercised compared to those who did not (3, $8 \%$ vs. $5.5 \%)$. In the logistic regression analysis, exercise was found to be associated with risk reduction in hypothyroidism, although no association in any direction was found between exercise and positive TPOAb. These results indicate that regular physical activity may be a protective factor for hypothyroidism. In our research we also found that subjects on weight watch had lower BMI as expected (22.686 \pm 3.037 vs. $22.961 \pm 3.463, \quad P=0.014)$, and lower prevalence of positive TOPAb and hypothyroidism (Table 3). However, there was no correlation between hyperthyroidism and weight watching or BMI. Many studies had reported that obesity can cause autoimmune disorders and increased TOPAb level, thus increases prevalence of hypothyroidism [28]. Our results were consistent with those reported in previous research.

\section{Daily staple, diet and salt intake}

Globally, iodine supplementation has been the primary method to prevent mental retardation caused by iodine deficiency. However, in the recent few decades, there was a concern that excessive iodine exposure in some individuals may increase the incidence of autoimmune thyroiditis. Some began to question mandatory iodization of salt. In our research, we found no difference in the prevalence of hyperthyroidism, hypothyroidism or positive TPOAb among subjects' groups with different amount of iodized salt consumption.

In this survey, we found an interesting relationship between subject's daily staple amount and their probability of developing TPOAb positive. It seemed that increased daily staple was correlated with lower risk of TPOAb positive (see Results section, Tables 3 and 4). Though the trend was moderate. The OR was 0.956 with $95 \%$ CI of $0.918-0.995$. It was unclear how daily staple affects thyroid disease development. However, several studies had indicated that starvation could cause hypothyroidism. It was shown that during long term energy withdrawal, thyroidal superoxide anion was able to lower the thyroid hormones thyroxine (T4) and triiodothyronine (T3) and cause a hypothyroid state in pigeons [29]. It was possible that decreased staple intake might raise TPOAb level, thus provoke the development of hypothyroidism.

\section{Stress}

It is well known that Stress was a provoking factor in the pathogenesis of Graves's disease. Stress may also precipitate patients who are predisposed to autoimmune thyroid dysfunction [30]. However, the relationship between stress and hypothyroidism remains obscure. Two previous studies conducted by Pan [31] and Rossana [32], respectively, did not find association between life events and the development of thyroid dysfunction. Many life style factors can evoke stress. Previous researches had detected insomnia [33] and chronic daily headache [34] as chronic stressors that can result in health problems. In our study, we evaluated potential association of these two stress factors with thyroid dysfunction. We found hyperthyroidism was less prevalent in people who suffered from insomnia than it was in people free of insomnia, although the prevalence for hypothyroidism was similar between the two groups. This was surprising as stress is known to trigger Graves Disease. However, it was possible that different types and degrees of stress affect thyroid function differently and insomnia-induced stress might be very different from those studied in previous research. For chronic daily headache, we found no association between headache and hyperthyroidism. However, prevalence of hypothyroidism was reduced in people who suffer from chronic daily headache $(5.7 \%$ vs. $3.2 \%)$. Again, the difference in the type and degree of stress investigated might explain the different observations in our study versus those of others. 


\section{Limitation of the study}

The study had certain limitations, including its lack of ability to infer causality due to the cross-sectional nature of the research design, as well as the use of a less stringent $p$ value to define statistical significance. Also, we used TSH level as the sole criteria for classifying thyroid dysfunction, which could potentially misclassify people with pituitary disease and resistance to thyroid hormone (though with very small probability) as having thyroid dysfunction. Further, the associations between lifestyle factors and blood TPOAb presentation were not analyzed under different thyroid disease status due to the design and scope of the study. Additional investigation would be needed to shed light on the complex interactions between lifestyle factors, TPOAb, and development of different thyroid diseases. Despite these limitations, the study provided useful epidemiological data for thyroid dysfunction research.

\section{Conclusion}

Within the Chinese She ethnic minority, we found associations between different lifestyle factors and the incidence of different thyroid diseases. Although some of the factors in this study may be Chinese She ethnic minority specific, the research provided much more information about the life style factors and the thyroid dysfunction. Continued accumulation of such epidemiological data is necessary towards a better understanding and management of thyroid dysfunction.

\section{Abbreviations \\ AITD: Autoimmune thyroid diseases; BMI: Body mass index; DBP: Diastolic blood pressure; HDL: High-density lipoprotein-cholesterol; HLA: Human leukocyte antigen; HR: Heart rate; LDL-c: Low-density lipoprotein-cholesterol; SBP: Systolic blood pressure; T3: Triiodothyronine; T4: Thyroid hormones thyroxine; TC: Total cholesterol; TG: Triglycerides; TPOAb: Thyroid peroxidase antibody; TSH: Thyroid Stimulating Hormone; UA: Uric acid}

\section{Acknowledgements}

The authors would like to thank community workers and patient advisers for participant recruitment and follow-up visits, Ningde Municipal Hospital of Fujian Medical University for allowing the study to run at their facility.

\section{Authors' contributions}

GC conceived, designed and developed the project. YLH, LCC, YYZ, JXP, LTL, $L Y Z$, and $L W$ assisted in data collection. $Y L H, L C C, Y Y Z$, JXP conducted data analysis. YLH developed the initial drafts of the manuscript. HBH, JPW helped in revising subsequent drafts. YLH, LCC and GC prepared the final draft of the manuscript. All authors read and approved the final manuscript.

\section{Funding}

This work was supported by grants C071002 for natural science foundation from Fujian province of China. The funding agency was not involved in the design of the study, collection, analysis, and interpretation of data, or preparation of the manuscript.

\section{Availability of data and materials}

The datasets used in the analyses described in this study are available from the corresponding author on reasonable request.

\section{Ethics approval and consent to participate}

Ethical clearance and approval were obtained from Ethics Committee of Fujian Medical University (Ethics Approval Reference Number 2009001). All study participants were informed about the purpose of the study and additional information was given as they need. Written informed consent was obtained from all participants. We had complied with the Declaration of Helsinki Ethical Principles for medical research involving human subjects [13].

\section{Consent for publication}

This manuscript does not report personal data such as individual details, images or videos; therefore, consent for publication is not applicable.

\section{Competing interests}

The authors declare that they have no competing interests.

\section{Author details}

'Department of Endocrinology and Metabolism, Zhongshan Hospital Xiamen University, 201-209 Hubin South Road, Xiamen 361004, China. ${ }^{2}$ Department of Endocrinology, Shengli Clinical Medical College of Fujian Medical University, Fuzhou 350001, China. ${ }^{3}$ Department of Geriatrics, Shengli Clinical Medical College of Fujian Medical University, Fuzhou 350001, China. ${ }^{4}$ Department of Endocrinology, Fujian Provincial Hospital, Fujian Academy of Medical Sciences, Fuzhou 350001, China.

Received: 6 February 2019 Accepted: 12 June 2019

Published online: 30 July 2019

\section{References}

1. Dorr M, Volzke H. Cardiovascular morbidity and mortality in thyroid dysfunction. Minerva Endocrinol. 2005;30(4):199-216.

2. Gulseren S, Gulseren L, Hekimsoy Z, Cetinay P, Ozen C, Tokatlioglu B. Depression, anxiety, health-related quality of life, and disability in patients with overt and subclinical thyroid dysfunction. Arch Med Res. 2006;37(1):133-9.

3. Gill PS, Patel JV, Chackathayil J, Webster C, Davis RC, Hughes E, Lip GY. Subclinical thyroid dysfunction and cardiac function amongst minority ethnic groups in the UK: a cross sectional study. Int I Cardiol. 2013;168(6):5218-20.

4. Effraimidis G, Wiersinga WM. Mechanisms in endocrinology: autoimmune thyroid disease: old and new players. Eur J Endocrinol. 2014;170(6):R241-52.

5. Wiersinga WM. Clinical relevance of environmental factors in the pathogenesis of autoimmune thyroid disease. Endocrinol Metab (Seoul). 2016;31(2):213-22.

6. Alegria-Torres JA, Baccarelli A, Bollati V. Epigenetics and lifestyle. Epigenomics. 2011;3(3):267-77.

7. Nystrom E, Bengtsson C, Lapidus L, Petersen K, Lindstedt G. Smoking--a risk factor for hypothyroidism. J Endocrinol Investig. 1993;16(2):129-31.

8. Vestergaard P. Smoking and thyroid disorders--a meta-analysis. Eur J Endocrinol. 2002;146(2):153-61.

9. Andersen SL, Olsen J, Wu CS, Laurberg P. Smoking reduces the risk of hypothyroidism and increases the risk of hyperthyroidism: evidence from 450,842 mothers giving birth in Denmark. Clin Endocrinol. 2014;80(2):307-14

10. Chen G, Wu J, Lin Y, Huang B, Yao J, Jiang Q, Wen J, Lin L. Associations between cardiovascular risk, insulin resistance, beta-cell function and thyroid dysfunction: a cross-sectional study in she ethnic minority group of Fujian Province in China. Eur J Endocrinol. 2010;163(5):775-82.

11. Lin Y, Lai X, Chen B, Xu Y, Huang B, Chen Z, Zhu S, Yao J, Jiang Q, Huang H, et al. Genetic variations in CYP17A1, CACNB2 and PLEKHA7 are associated with blood pressure and/or hypertension in she ethnic minority of China. Atherosclerosis. 2011;219(2):709-14.

12. XuY LY, Chen G, Huang B, Chen Z, Yao L, Chen Z. Glycated hemoglobin, diabetes mellitus, and cardiovascular risk in a cross-sectional study among she Chinese population. J Endocrinol Invest. 2012;35(1):35-41.

13. World Medical Association Declaration of Helsinki, et al. Jama. 2000;284(23):3043-5.

14. Shan Z, Chen L, Lian X, Liu C, Shi B, Shi L, Tong N, Wang S, Weng J, Zhao J, et al. lodine status and prevalence of thyroid disorders after introduction of mandatory universal salt iodization for 16 years in China: a cross-sectional study in 10 cities. Thyroid. 2016;26(8):1125-30. 
15. Endocrinol EJ. Prevalence of thyroid disease, thyroid dysfunction and thyroid peroxidase antibodies in a large, unselected population. The Health Study of Nord-Trondelag (HUNT). Eur J Endocrinol. 2000;143(5):639-47.

16. Holm IA, Manson JE, Michels KB, Alexander EK, Willett WC, Utiger RD. Smoking and other lifestyle factors and the risk of Graves' hyperthyroidism. Arch Intern Med. 2005;165(14):1606-11.

17. Carle A, Bulow Pedersen I, Knudsen N, Perrild H, Ovesen L, Rasmussen LB, Jorgensen T, Laurberg P. Graves' hyperthyroidism and moderate alcohol consumption: evidence for disease prevention. Clin Endocrinol. 2013;79(1):111-9.

18. Carle A, Pedersen IB, Knudsen N, Perrild H, Ovesen L, Rasmussen LB, Jorgensen T, Laurberg P. Moderate alcohol consumption may protect against overt autoimmune hypothyroidism: a population-based case-control study. Eur J Endocrinol. 2012;167(4):483-90.

19. Takvorian SU, Merola JF, Costenbader KH. Cigarette smoking, alcohol consumption and risk of systemic lupus erythematosus. Lupus. 2014;23(6):537-44.

20. Wiersinga WM. Smoking and thyroid. Clin Endocrinol. 2013;79(2):145-51.

21. Belin RM, Astor BC, Powe NR, Ladenson PW, et al. Smokers have high serum antithyroid antibody and thyrotropin concentrations less often and low serum thyrotropin concentrations more often than nonsmokers. J Clin Endocrinol Metab. 2004:89:6077-86

22. CH CNH, Kim KW, Kim HL, Lee SY, Choi SH, Lim S, Park YJ, Park DJ, Jang HC, Cho BY. Interaction between cigarette smoking and iodine intake and their impact on thyroid function. Clin Endocrinol. 2010;73:264-70.

23. ZR WDW, Yao YM, Zhu XM, Yin YM, Zhao GJ, Dong N, Sheng ZY. Stimulation of a7 nicotinic acetylcholine receptor by nicotine increases suppressive capacity of naturally occurring CD4+CD25+ regulatory $T$ cells in mice in vitro. J Pharmacol Exp Ther. 2010;335:553-61.

24. Arnson $Y$, Shoenfeld $Y$, Amital $H$. Effects of tobacco smoke on immunity, inflammation and autoimmunity. J Autoimmun. 2010;34(3):J258-65.

25. Sawicka-Gutaj N, Gutaj P, Sowinski J, Wender-Ozegowska E, Czarnywojtek A, Brazert J, Ruchala M. Influence of cigarette smoking on thyroid gland--an update. Endokrynol Pol. 2014;65(1):54-62.

26. Ciloglu F, Peker I, Pehlivan A, Karacabey K, Ilhan N, Saygin O, Ozmerdivenli R. Exercise intensity and its effects on thyroid hormones. Neuro Endocrinol Lett. 2005;26(6):830-4.

27. Arkader R, Rosa MR, Moretti G. Physiological changes of Exercise of Thermogenesis, Thyroid Homeostasis and Inflammations. Endocrinol Metabol Int J. 2016;4(5):1-5

28. Dahl M, Ohrt JD, Fonvig CE, Kloppenborg JT, Pedersen O, Hansen T, Holm JC. Subclinical hypothyroidism in Danish lean and obese children and adolescents. J Clin Res Pediatr Endocrinol. 2016:8-16.

29. Prem P, Parihar MS, Malini L, Pradeep KG. Starvation induced hypothyroidism involves perturbations in thyroid superoxide-SOD system in pigeons. Biochem Mol Biol Int. 1998;45(1):73-83.

30. Kung AW. Life events, daily stresses and coping in patients with Graves' disease. Clin Endocrinol. 1995:42(3):303-8.

31. Pan RM-D. Triggering role of stress and pregnancy in the occurrence of 98 cases of Graves' disease compared to 95 cases of Hashimoto thyroiditis and 97 cases of thyroid modules. Annales d Endocrinologie. 1998;59(2):107-12.

32. Oretti RG, Harris B, Lazarus JH, Parkes AB, Crownshaw T. Is There an Association between Life Events, Postnatal Depression and Thyroid Dysfunction in Thyroid Antibody Positive Women? Int I Soc Psychiatry. 2003:49(1):70-6.

33. Vgontzas AN, Tsigos C, Bixler EO, Stratakis CA, Zachman K, Kales A, VelaBueno A, Chrousos GP. Chronic insomnia and activity of the stress system: a preliminary study. J Psychosom Res. 1998;45(1):21-31.

34. Galego JC, Moraes AM, Cordeiro JA, Tognola WA. Chronic daily headache: stress and impact on the quality of life. Arq Neuropsiquiatr. 2007;65(4B):1126-9.

\section{Publisher's Note}

Springer Nature remains neutral with regard to jurisdictional claims in published maps and institutional affiliations.

Ready to submit your research? Choose BMC and benefit from:

- fast, convenient online submission

- thorough peer review by experienced researchers in your field

- rapid publication on acceptance

- support for research data, including large and complex data types

- gold Open Access which fosters wider collaboration and increased citations

- maximum visibility for your research: over $100 \mathrm{M}$ website views per year

At BMC, research is always in progress.

Learn more biomedcentral.com/submissions 